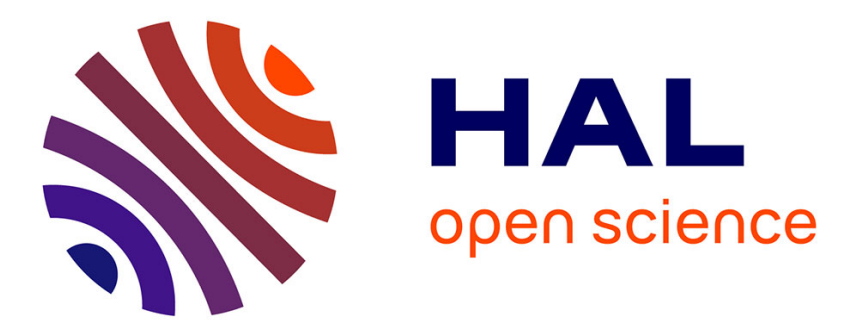

\title{
Single-shot time resolved study of the photo-reversible phase transition induced in flakes of Ti3O5 nanoparticles at room temperature
}

\author{
Amine Ould-Hamouda, Hiroko Tokoro, Shin-Ichi Ohkoshi, Eric Freysz
}

\section{- To cite this version:}

Amine Ould-Hamouda, Hiroko Tokoro, Shin-Ichi Ohkoshi, Eric Freysz. Single-shot time resolved study of the photo-reversible phase transition induced in flakes of Ti3O5 nanoparticles at room temperature. Chemical Physics Letters, 2014, 608, pp.106-112. 10.1016/j.cplett.2014.05.073 . hal-01063977

\section{HAL Id: hal-01063977 \\ https://hal.science/hal-01063977}

Submitted on 26 Feb 2015

HAL is a multi-disciplinary open access archive for the deposit and dissemination of scientific research documents, whether they are published or not. The documents may come from teaching and research institutions in France or abroad, or from public or private research centers.
L'archive ouverte pluridisciplinaire HAL, est destinée au dépôt et à la diffusion de documents scientifiques de niveau recherche, publiés ou non, émanant des établissements d'enseignement et de recherche français ou étrangers, des laboratoires publics ou privés.

\section{()ㅜ(1)}

Distributed under a Creative Commons Attribution - NonCommerciall 4.0 International 


\title{
Single-shot time resolved study of the photo-reversible phase transition induced in flakes of $\mathrm{Ti}_{3} \mathrm{O}_{5}$ nanoparticles at room temperature
}

\author{
A. Ould-Hamouda ${ }^{\mathrm{a}, \mathrm{b}}$, H. Tokoro ${ }^{\mathrm{c}}$, S.-I. Ohkoshi ${ }^{\mathrm{c}}$, E. Freysz ${ }^{\mathrm{a}, \mathrm{b}, *}$ \\ a Univ. de Bordeaux, LOMA, UMR 5798, 351 Cours de la Libération, 33405 Talence Cedex, France \\ ${ }^{\mathrm{b}}$ CNRS, LOMA, UMR 5798, 351 Cours de la Libération, 33405 Talence Cedex, France \\ ${ }^{\mathrm{c}}$ Department of Chemistry, School of Science, The University of Tokyo, 7-3-1 Hongo, Bunko-ku, Tokyo 113-0033, Japan
}

\begin{abstract}
A B S T R A C T
We have performed single-shot time-resolved reflectivity measurements of the photo-reversible phase transition between $\lambda$ - and $\beta$-phases of $\mathrm{Ti}_{3} \mathrm{O}_{5}$ nanoparticles at room temperature. These phase transitions are induced using nanosecond and millisecond laser pulses and are revealed by Raman spectroscopy. Upon nanosecond pulsed excitation, the $\lambda$ - to $\beta$-phase transition is detected in about $900 \mathrm{~ns}$, whereas the $\beta$ - to $\lambda$-phase transition is noticed in less than $20 \mathrm{~ns}$. The $\lambda$ - to $\beta$-phase transition induced by quasi continuous wave laser happens on the millisecond time scale. We propose a scenario that accounts very well for the observed phenomena.
\end{abstract}

\section{Introduction}

Materials in which phase-change can be photo-induced offer many prospective for the development of high density optical memory devices. Important researches are pursued in this field. To date photoinduced phase transitions have been reported in different materials: chalcogenide [1,2], spin crossover complexes [3-6] and photochromic compounds [7-9], cyanobridged metal assemblies [10-16], donor-acceptor stacked molecules [17, 18], perovskite oxide [19]. Very recently, a photoreversible metal-semiconductor phase transition has been recorded at room temperature within a new phase of $\mathrm{Ti}_{3} \mathrm{O}_{5}$ [20]. It results from a unique metallic phase of titanium oxide $\lambda-\mathrm{Ti}_{3} \mathrm{O}_{5}$ that is trapped within the nanoparticles. At room temperature, light irradiation causes a phase transformation between this $\lambda-\mathrm{Ti}_{3} \mathrm{O}_{5}$ metastable phase and the conventional semiconductor $\beta-\mathrm{Ti}_{3} \mathrm{O}_{5}$ phase. This economical and environmentally benign material offers interesting prospects since it makes it possible to achieve up to 1 terabit inch ${ }^{-2}$ memory density using near field light. Different mechanisms have been proposed to account for these photo-induced phase transitions. All are triggered by absorption of the laser energy by the nanoparticles [20]. The thermodynamic analysis suggests that the laser induce $\lambda$-to $\beta$-phase transition results from a transition of the metastable state $\left(\lambda-\mathrm{Ti}_{3} \mathrm{O}_{5}\right)$ to a truly stable state $\left(\beta-\mathrm{Ti}_{3} \mathrm{O}_{5}\right)$. For the reverse photo-induced phase transition, it was suggested that once excited

* Corresponding author at: Univ. de Bordeaux, LOMA, UMR 5798, 351 Cours de la Libération, 33405 Talence Cedex, France. Fax: +33 540006970.

E-mail address: e.freysz@loma.u-bordeaux1.fr (E. Freysz). in the conduction band of the $\beta-\mathrm{Ti}_{3} \mathrm{O}_{5}$, the excited state drives directly the sample to $\lambda-\mathrm{Ti}_{3} \mathrm{O}_{5}$ during the pulsed laser irradiation or, when continuous wave excitation is used, photothermally to $\lambda-\mathrm{Ti}_{3} \mathrm{O}_{5}$ through $\alpha-\mathrm{Ti}_{3} \mathrm{O}_{5}$ (that is, $\beta-\mathrm{Ti}_{3} \mathrm{O}_{5} \rightarrow \alpha-\mathrm{Ti}_{3} \mathrm{O}_{5} \rightarrow \lambda-\mathrm{Ti}_{3} \mathrm{O}_{5}$ ) [20]. These different mechanisms have not yet been confirmed. Moreover, the time scales over which these phase transitions are taking place have not been measured. Determination of these time scales is crucial for practical applications and it can reveal the physical mechanisms that are driving these phase transitions. To address this problem, we have performed single-shot time resolved reflectivity measurements using flakes of $\mathrm{Ti}_{3} \mathrm{O}_{5}$ nanoparticles. The sample was excited by nanosecond pulse or Quasi Continuous Wave $(\mathrm{QCW})$ and the variation of its reflectivity was measured over time scales ranging from 20 ns to $1 \mathrm{~s}$. To reveal the photo-induced phase transitions, we used Raman spectroscopy. The analysis of our data demonstrates that under nanosecond pulsed excitation, the $\lambda-\mathrm{Ti}_{3} \mathrm{O}_{5}$ to $\beta-\mathrm{Ti}_{3} \mathrm{O}_{5}$ and the $\beta-\mathrm{Ti}_{3} \mathrm{O}_{5}$ to- $\lambda-\mathrm{Ti}_{3} \mathrm{O}_{5}$ phase transitions occur for different pump fluences and on very different time scales. Upon nanosecond pulsed excitation, the $\lambda$ - to $\beta$-phase transition is detected after $\sim 900 \mathrm{~ns}$, whereas the $\beta$ - to $\lambda$-phase transition is recorded in less than $20 \mathrm{~ns}$. Under QCW excitation, the $\lambda$ - to $\beta$-phase transition happens on the millisecond time scale. This ensemble of data makes it possible to propose a scenario that accounts very well for the recorded phenomena.

\section{Sample preparation and single-shot experimental set-up}

The sample preparation has been presented elsewhere [20]. The metastable phase of $\lambda-\mathrm{Ti}_{3} \mathrm{O}_{5}$ can be obtained in two morphological 


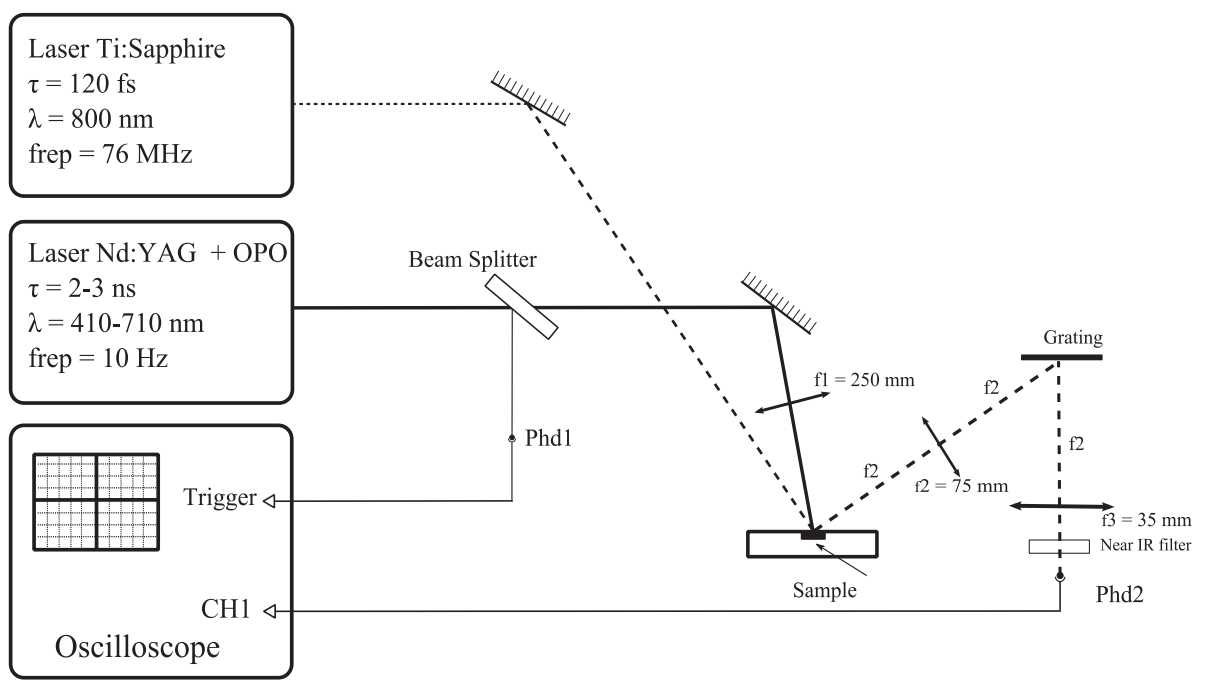

Figure 1. All optical single-shot pump-probe reflectivity measurement set-up.

types by different synthetic methods [20]. The flakes $(2 \pm 0.5 \mu \mathrm{m})$ of the $\lambda-\mathrm{Ti}_{3} \mathrm{O}_{5}$ are assembled from $25 \pm 15 \mathrm{~nm}$ nanocrystals. They were prepared by calcinations of anatase form of $\mathrm{TiO}_{2}$ nanoparticles $(7 \mathrm{~nm})$ under hydrogen with a flow speed of $0.3 \mathrm{dm}^{-3} \mathrm{~min}^{-1}$ at $1200{ }^{\circ} \mathrm{C}$ for two hours. X-ray diffraction (XRD) pattern of $\lambda \mathrm{Ti}_{3} \mathrm{O}_{5}$ nanoparticles indicates a crystalline structure of $C 2 / \mathrm{m}$ symmetry class. XRD measurements have also shown that, as the temperature increases, the diffraction peaks of the $\lambda-\mathrm{Ti}_{3} \mathrm{O}_{5}$ continuously changed to $\alpha-\mathrm{Ti}_{3} \mathrm{O}_{5}$ peaks with a crystal structure of $\mathrm{Cmcm}$ symmetry class. Moreover, heating the sample to $640 \mathrm{~K}$ and then cooling it to $300 \mathrm{~K}$ caused $\alpha-\mathrm{Ti}_{3} \mathrm{O}_{5}$ to return to $\lambda-\mathrm{Ti}_{3} \mathrm{O}_{5}$. This measurements as well as a meaningful peak recorded during differential scanning calorimetry experiments make it possible to propose to classify this latter phase transition as a second order phase transition [20]. With that respect, it is important to mention that for a single-crystal a $\beta-\mathrm{Ti}_{3} \mathrm{O}_{5}$ to $\alpha-\mathrm{Ti}_{3} \mathrm{O}_{5}$ first-order phase transition is recorded around $470 \mathrm{~K}$. In the experiments reported hereafter, the flake forms of $\lambda-\mathrm{Ti}_{3} \mathrm{O}_{5}$ were compacted into a pellet using a manual hydraulic press with a pressure of about $0.1 \mathrm{GPa}$. To be sure that the pellet was set in $\lambda-\mathrm{Ti}_{3} \mathrm{O}_{5}$ phase, it was further heated at $640 \mathrm{~K}$ for $30 \mathrm{~min}$ and cooled at room temperature.

Our all optical single-shot pump-probe set-up is sketched in Figure 1, its working principle has been described elsewhere [21]. The pulses issued from a nanosecond Optical Parametric Oscillator (OPO) (Panther from Continuum) pumped by a frequency tripled nanosecond Nd:YAG laser (Surelite II-10 from Continuum) are used to excite the sample. This OPO delivers, at a maximal repetition rate of $10 \mathrm{~Hz}$, visible pulses that can be tuned in a spectral domain ranging from $410 \mathrm{~nm}$ to $710 \mathrm{~nm}$. The pulse duration was found to change versus the wavelength in between 2 ns and 3 ns. These pulses were slightly focused on the sample and their energies were controlled thanks to a variable neutral density filter. A Ti: Sapphire oscillator (Mira, Coherent) generates the probe beam. It delivers, at a repetition rate of $76 \mathrm{MHz}$, near infrared (centered at $\sim 800 \mathrm{~nm}$ ) femtosecond pulses (pulse duration $\sim 120 \mathrm{fs}$ ) with an energy of $\sim 10 \mathrm{~nJ}$. During the experiment, the probe pulse energy is set to $0.5 \mathrm{~nJ}$ and the probe beam size is reduced by a factor of two on the sample surface compared to the pump beam. The probe light diffused by the sample is collimated by a lens, diffracted by a holographic grating (1200 grooves $\mathrm{mm}^{-1}$ ) blazed at $800 \mathrm{~nm}$, collected by a lens and focused on the small detecting area of a fast photodiode (DET-10A; Thorlabs). A colored filter with a high (resp. very low) transmission above $800 \mathrm{~nm}$ (resp. below $600 \mathrm{~nm}$ ) is inserted in front of the photodiode. Upon excitation, the photodiode produces electrical pulses having a rising time of $\sim 1$ ns. These pulses are sampled, up to 5 Giga-samples/s, by a sampling oscilloscope having a bandwidth of $1 \mathrm{GHz}$ and 8 Mega-bits per channel memory length (Tektronix, TDS 5104). The set-up works as follow. When the pump pulse, delivered on demand, is shining on the sample, it triggers the oscilloscope that records during up to $1 \mathrm{~s}$, the Ti:Sapphire pulses reflected by the sample. This reflectivity measurement makes it possible to record the evolution of the properties of the sample during the phase transitions. In practice, the pulse repetition rate of Ti:Sapphire oscillator $(\sim 76 \mathrm{MHz})$ limits our experimental resolution to $\sim 13 \mathrm{~ns}$.

\section{Experimental results}

We have carried out different experiments. At first, the sample was excited by the nanosecond pulses centered at $532 \mathrm{~nm}$ and, after each excitations; we watched its evolution using a small magnification microscope. For high pump fluence $\left(1.310^{-5} \mathrm{~mJ} \mu \mathrm{m}^{-2}\right)$, the color of the irradiated area changed from black to brown (Figure 2a and $b$ ). Thanks to XRD, this area has been shown to be a $\beta$-phase of $\mathrm{Ti}_{3} \mathrm{O}_{5}$ [20]. Once in the $\beta$-phase, we noticed that the $\beta$ - to- $\lambda$-phase transition can be achieved using a sequence of four or five pump pulses with lower fluence $\left(5.710^{-6} \mathrm{~mJ} \mu \mathrm{m}^{-2}\right)$. The photo-excited area is then switched from brown to dark black (Figure 2c). Here again and thanks to XRD, it has been previously shown that the black area was in the $\lambda$-phase. The latter area can be further photo-switched from black to brown (Figure 2d). To check the state of the virgin and irradiated sample, we decided to perform Raman spectroscopy. The Raman spectra were collected using a Thermo Scientific DXR Raman microscope. The Raman spectrum of the sample in the $\beta$-phase of $\mathrm{Ti}_{3} \mathrm{O}_{5}$ is in good agreement with previously published data [22]. To the best of our knowledge the Raman spectra of the $\lambda$-phase has not been previously reported. The spectra of the sample before and after irradiation, in $\lambda$ - and $\beta$-phase, are presented in Figure 3. They reveal that (i) after the photo-induced phase transitions marked Raman shift can be recorded (ii) after a $\lambda$ - to $\beta$-phase transition followed by a $\beta$ - to- $\lambda$-phase transition, the sample recovers its initial Raman spectrum. These measurements indicate that Raman spectroscopy is a very convenient mean to reveal such photoinduced phase transition.

In a second step, using the single-shot reflectivity set-up, we have recorded the kinetics of the switching between the $\lambda$ - and $\beta$-phases for fixed value of the pump fluence of $1.310^{-5} \mathrm{~mJ} \mu \mathrm{m}^{-2}$ 

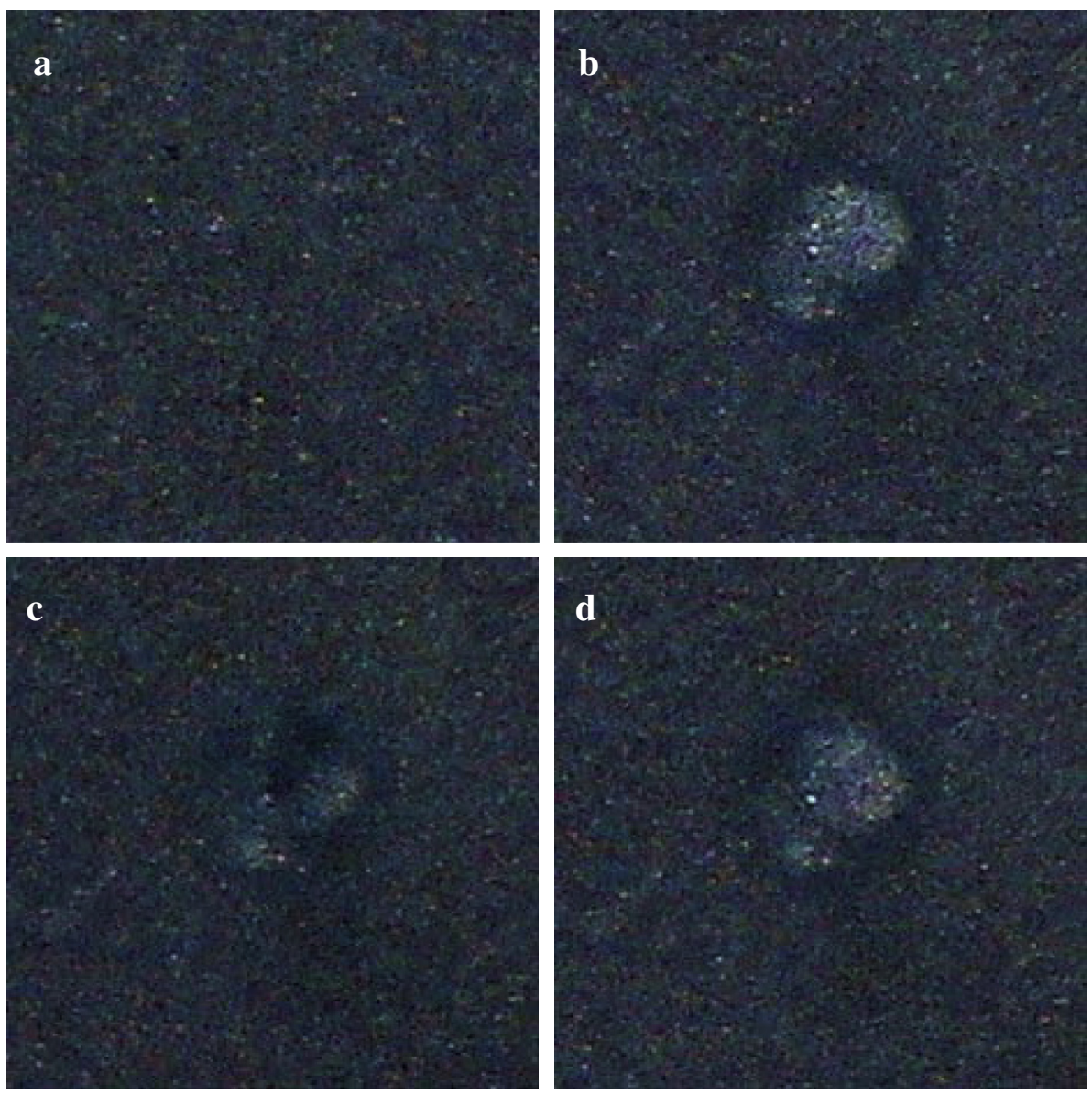

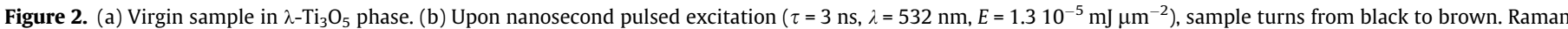

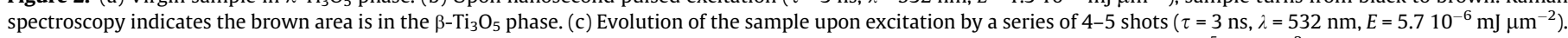

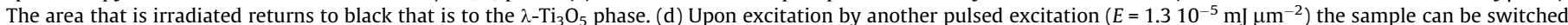
again to $\beta-\mathrm{Ti}_{3} \mathrm{O}_{5}$ phase. (For interpretation of the references to colour in this figure legend, the reader is referred to the web version of this article.)

that wavelength was fixed at $532 \mathrm{~nm}$. Prior to these experiments, we measured the reflectivity of the sample in the $\beta$ - and $\lambda$-phases. It increases by $\sim 50 \%$ when going from the $\lambda$ - to the $\beta$-phase. A typical evolution of the reflectivity signal, named $S_{p}+$ pr hereafter, is displayed in Figure 4a. Upon excitation, $S_{p}+$ pr $(t)$ increases almost instantaneously and then relaxes slowly. Over this general trend, the electric signal produced by each probe pulses is superposed (inset in Figure 4a). Surprisingly, we have also recorded a signal when the probe pulse is switched off. This signal, named $S_{p}(t)$, was similar in the $\beta$ - and $\lambda$-phases and it is displayed in Figure $4 a$ (blue line). It is neither due to light diffusion of the pump pulse nor related to a saturation of the fast photodiode [23]. It is likely due to laser induced plasma: the laser pulse vaporized a very tiny amount of the sample; inducing very hot plasma that radiates in the near infrared range. In agreement with this hypothesis, it is interesting to mention that wearing laser safety goggles at the excitation wavelength; we were able to watch a very weak white flash associated to the formation of plasma upon laser excitation. To retrieve the actual reflectivity evolution of the sample related to the $\lambda$ - to $\beta$-phase transition, we computed $S_{p}(t)-S_{p+p r}(t)$ and plotted its evolution (Figure $4 \mathrm{~b}$ ). We found that the relaxation of the latter signal can be well fitted considering a background plus a single exponential decay law. The fit of these relaxation signals indicates that the maximum (red-doted lines in Figure 4b), minimum (red dotted lines in Figure $4 \mathrm{~b}$ ) and averaged value of the reflectivity change (red solid lines in Figure 4 b) reached their steady state values with a constant time of $900 \pm 20$ ns.
For the $\mathrm{Ti}_{3} \mathrm{O}_{5}$ sample in the $\beta$-phase, we reduced the pulse fluence to $5.710^{-6} \mathrm{~mJ} \mu \mathrm{m}^{-2}$ and recorded the evolution of the reflectivity of the sample upon excitation by a sequence of pulses (Figure 5). Upon excitation by the first and second pump pulse, the steady state reflectivity of the sample decreases by $\sim 40 \pm 10 \%$ and $20 \pm 10 \%$ respectively. The excitation of the sample by a third and a fourth pump pulse has no further impact. In fact, after the second pump pulse, the sample has almost reached the reflectivity expected when it is in the $\lambda$-phase. Figures $5 c-d$ display the signal recorded for the third and forth pump pulse with (Figure $5 \mathrm{c}$ ) and without (Figure $5 d$ ) the probe pulses respectively. On Figure $5 d$, one can notice the presence of a very weak signal that lasts few nanoseconds close to zero time delay. It is probably this signal that is responsible for the weak reflectivity change displayed on Figure $5 c$. The fit of the signals displayed in Figure $5 a-b$ (considering a background and a single exponential decay) indicates that the maximum (red-dotted lines), minimum (red dotted lines) and averaged value (red solid lines) of the reflectivity change reached its steady state with a constant time of $20 \pm 5 \mathrm{~ns}$.

We have also switched our sample from the $\beta$ - to $\lambda$-phase using QCW excitation. In the latter case a mechanical shutter, set in front of a continuous laser working at $532 \mathrm{~nm}$ (Coherent, Verdi laser), was switched on for $2 \mathrm{~ms}$. The change of the reflectivity of the sample upon such an excitation with a power density of $2.410^{-3}$ $\mathrm{mW} \mu \mathrm{m}^{-2}$ is displayed in Figure 6a. One can notice that the reflectivity of the sample decreases when the excitation is stopped. It was found to decrease exponentially with a time constant of 

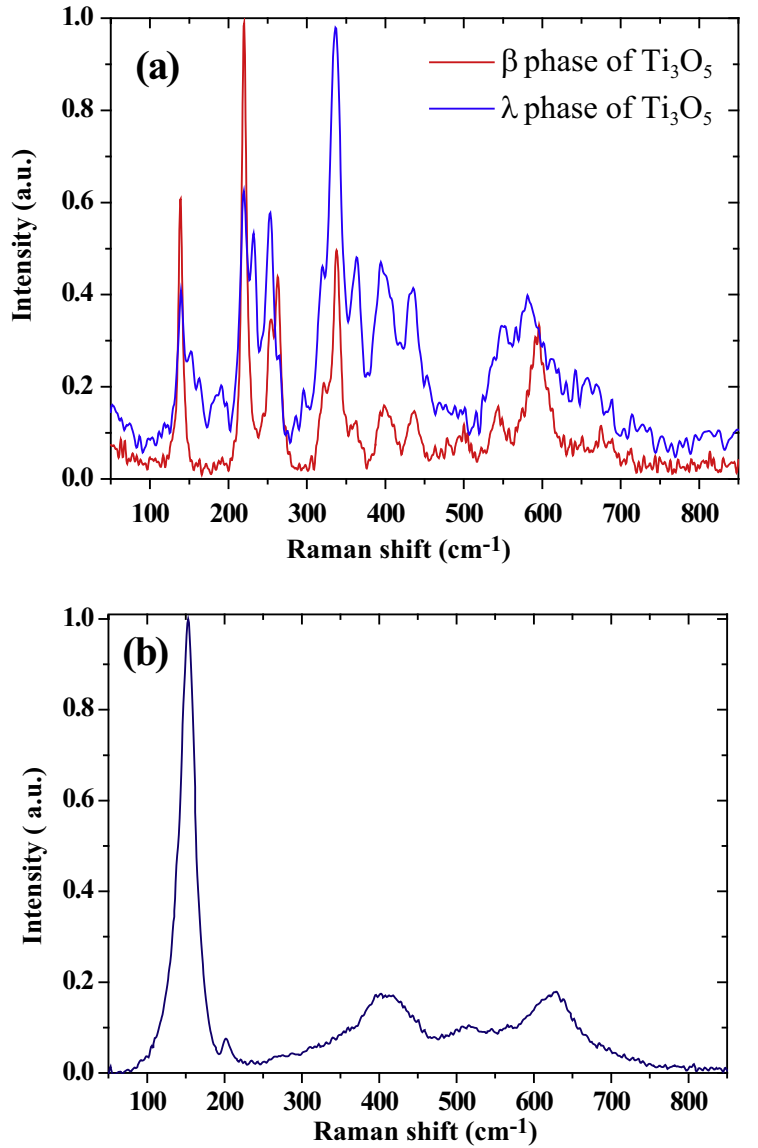

Figure 3. (a) Raman spectra of the sample in the $\lambda$ - and $\beta-\mathrm{Ti}_{3} \mathrm{O}_{5}$ phases. (b) Raman spectrum of the $\lambda-\mathrm{Ti}_{3} \mathrm{O}_{5}$ nanoparticles after $\mathrm{QCW}$ laser excitation $\left(I=2.810^{-3}\right.$ $\mathrm{mW} \mu \mathrm{m}^{-2}$ ). This spectrum resembles the one recorded for $\mathrm{TiO}_{2}$ nanoparticles.

$1.5 \pm 0.2 \mathrm{~ms}$ (solid line in Figure 6a). The Raman spectrum of the photo-excited area indicates the sample is in the $\lambda$-phase. We could switch back the sample from the $\lambda$ - to $\beta$-phase exciting the sample with a nanosecond pulse centered at $532 \mathrm{~nm}$. We have performed the same experiment increasing the intensity of the pump beam to $2.810^{-3} \mathrm{~mW} \mu \mathrm{m}^{-2}$. After its excitation, the reflectivity of the sample was found to increase (Figure 6b). However, the photoexcited area turned from black to white. The Raman spectrum of this area resembles to Raman spectrum of $\mathrm{TiO}_{2}$ nanoparticles (Figure 3b) [24]. This area remains as it is upon excitation by subsequent nanosecond pulses.

\section{Discussion}

The recorded kinetics indicate that upon excitation by nanosecond pulses the change of the reflectivity is not instantaneous. During the $\lambda$ - to $\beta$-phase transition, it takes $\sim 900 \mathrm{~ns}$ for the reflectivity to reach a steady state whereas during the transition from $\beta$ - to $\lambda$ phase, this happens on $\sim 20$ ns a much shorter time scale. Since these phase transitions are photo-induced and since both $\lambda$ - and $\beta$-phase absorb at the laser light, one must consider photothermal effects [20]. The energy $Q$ absorbed by the flake reads $Q \sim S \alpha E$ where $S, \alpha$ and $E$ are the surface of the flake, the percentage of energy absorbed and the fluence of the laser pulse respectively. The latter induces an increase of the temperature of the flakes that reads: $\Delta T \sim Q /\left(V \rho C_{p}\right)$ where $V, \rho$ and $C_{p}$ are respectively the volume, the density and the heat capacity of these particles. Even if most of these latter parameters are unknown, it is interesting to evaluate the increase in temperature of the sample $\Delta T$ upon excitation by a nanosecond pulse. The absorption coefficient of the $\lambda-\mathrm{Ti}_{3} \mathrm{O}_{5}$ flake is estimated to be in between $5 \%$ and $10 \%$. Then considering that the density and the heat capacity of our sample is roughly similar to that of bulk $\beta-\mathrm{Ti}_{3} \mathrm{O}_{5}\left(\rho \sim 4.6 \times 10^{3} \mathrm{~kg} \mathrm{~m}^{-3}\right.$ and $C_{p} \sim 520 \mathrm{~J} \mathrm{~kg}^{-1} \mathrm{~K}^{-1}$ ), one can easily show that for a pump pulse fluence of $\sim 10^{-5} \mathrm{~mJ} \mathrm{~m}^{-2}$ and for the typical size distribution of the flakes (radius $\sim 1 \mu \mathrm{m}$ ), the temperature increase $\Delta T$ upon laser excitation ranges in between $313 \mathrm{~K}$ and $627 \mathrm{~K}$. However, since the absorption coefficient of the flakes is quite large, most of the pump energy is dumped on a very thin layer at the surface of these particles. The thickness of the layer should be about ten times smaller than the radius of the particles (i.e. $\sim 0.2 \mu \mathrm{m}$ ). Hence, upon laser excitation, the laser energy is only released over a very small volume of the flake and the actual increase of temperature is more important, and is in between $1000 \mathrm{~K}$ and $2000 \mathrm{~K}$. As a consequence, the sample surface is almost instantaneously driven at a temperature where a $\lambda$ - to $\alpha-\mathrm{Ti}_{3} \mathrm{O}_{5}$ phase transition can take place. Moreover, such a temperature accounts very well for the laser induced plasma. Indeed, a very high thermal gradient is produces near
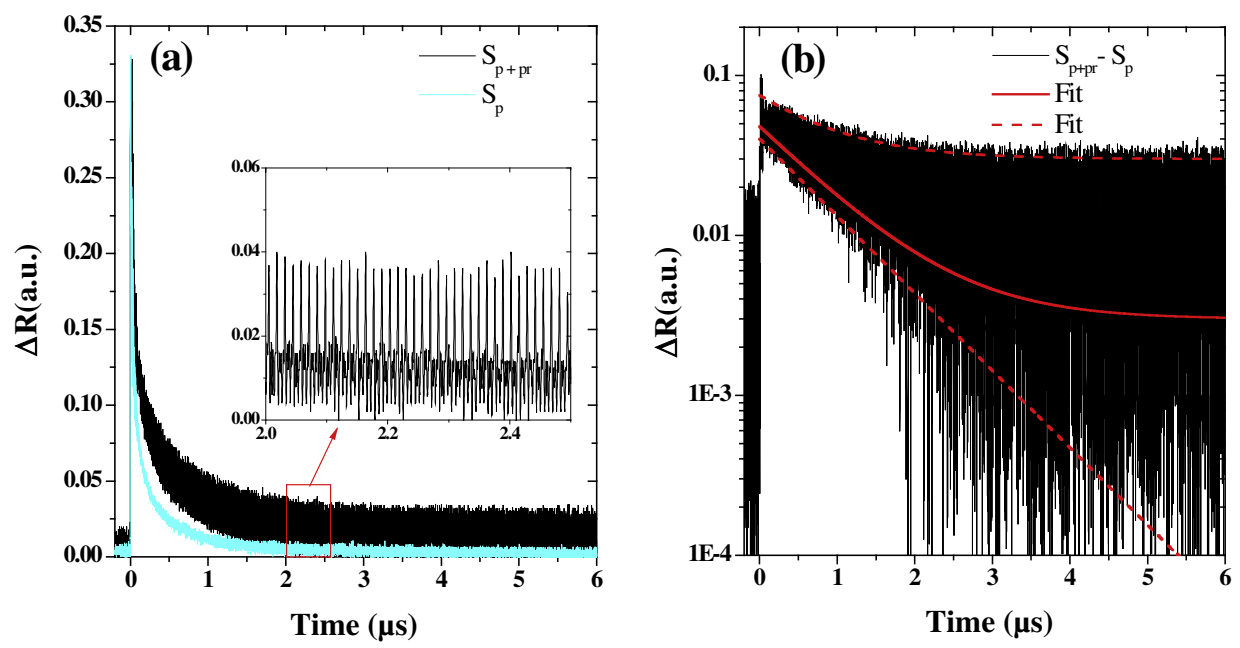

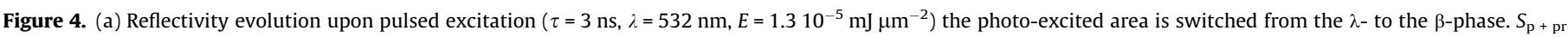

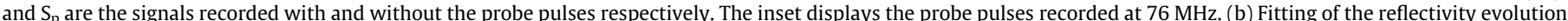

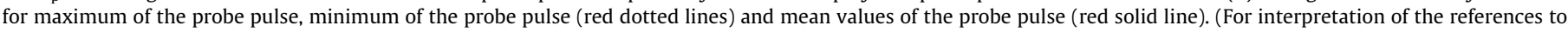
colour in this figure legend, the reader is referred to the web version of this article.) 

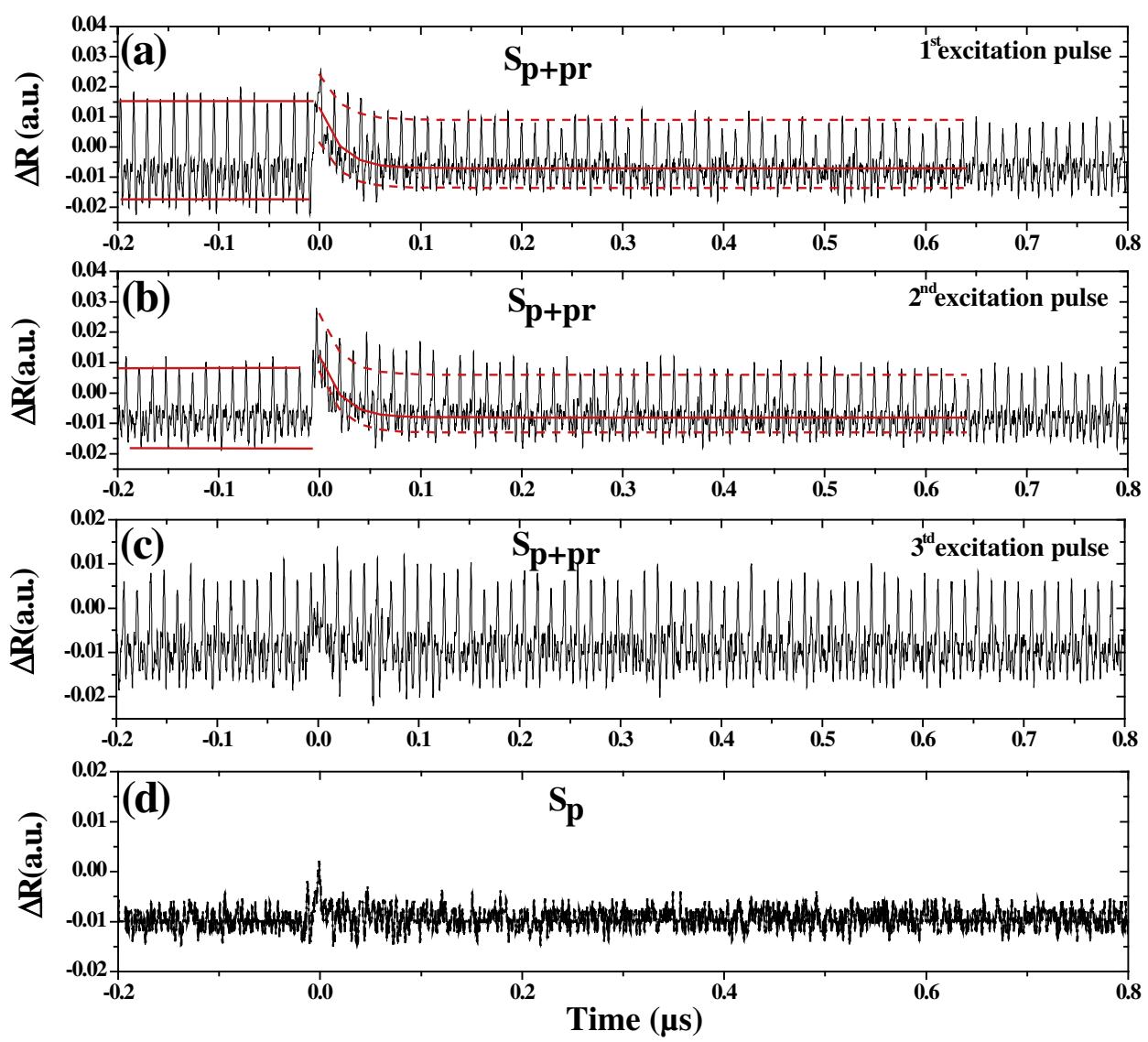

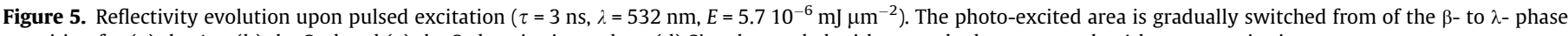
transition for (a) the 1st, (b) the 2nd and (c) the 3rd excitation pulses. (d) Signal recorded without probe beam upon the 4th pump excitation.

the surface of the particles that induces a shock wave that propagates with about the speed of sound $\left(v>10^{3} \mathrm{~m} \mathrm{~s}^{-1}\right)$. During this process a tiny amount of the sample is also ejected from the surface. This latter partly absorbs the pump laser pulse and produces a very hot plasma. The plasma radiates in the visible range and it is responsible for the signal recorded without the probe beam. It is interesting to also mention that it has been theoretically shown that the temperature required to induce $\lambda$ - to $\beta-\mathrm{Ti}_{3} \mathrm{O}_{5}$ phase transition is drastically reduced as the pressure increases within the particle [25]. Therefore, as the surface of the flake is heated and turned to the $\alpha-\mathrm{Ti}_{3} \mathrm{O}_{5}$, the laser-induced shock wave turns, at least partly, the surface of the sample from $\alpha$ - to $\beta-\mathrm{Ti}_{3} \mathrm{O}_{5}$. Hence, we can consider that upon its excitation, the surface of the sample is largely seeded by $\beta-\mathrm{Ti}_{3} \mathrm{O}_{5}$ phase domains. After its excitation, the heat deposited on the sample is partly thermally radiated and partly diffused towards its center. These two processes as well as the relaxation of the induced plasma are responsible for the increase and the rapid decrease of the reflectivity of the sample without the probe pulses. Now, as the heat deposited at the surface of the particles diffuses, the temperature at the center of the particle increases. Considering the thermal diffusivity of the sample is about that of bulk $\mathrm{TiO}_{2}\left(D_{t h} \sim 410^{-6} \mathrm{~m}^{2} \mathrm{~s}^{-1}\right)$ and neglecting the energy radiated by the surface of the sample, one can readily compute that the center of the particle reach a temperature in between $600 \mathrm{~K}$ and $1200 \mathrm{~K}$ in $\sim 6 \mathrm{~ns}$. Since the latter temperatures are above the $\alpha$ - to $\beta-\mathrm{Ti}_{3} \mathrm{O}_{5}$ phase transition, such a phase transition is likely to take place. According to this scenario, as heat diffuses, the seeds of $\beta-\mathrm{Ti}_{3} \mathrm{O}_{5}$ phase produced at the surface of the particle grow and propagate towards the center of the particle. One should also mention that according to this scenario and in agreement with previous observations [20], a pump fluence threshold is required to heat the surface of the sample above the $\lambda$ - to $\beta$-phase transition. However, to further validate this scenario one must address the following question: Why does it take $\sim 900$ ns for the whole particle to switch from $\lambda$ - to $\beta$-phase? To answer this question one should mention that, in a single crystal, an $\alpha-\mathrm{Ti}_{3} \mathrm{O}_{5}$ to $\beta-\mathrm{Ti}_{3} \mathrm{O}_{5}$ first order phase transition is recorded at about $480 \mathrm{~K}$ (Figure 7). Therefore, as long as the $\alpha$ - to $\beta$-phase transition is taking place within the flakes, some heat is released and can compensate for the diffusion of heat from the surface to the center of the flake. Hence, one should consider that the 900 ns time scale is the time it takes for the excess of heat to be released from the flake.

A similar process can also account for the $\beta$ - to $\lambda$-phase transition. In this case, two important points have to be stressed: the pump pulse fluence and the absorption of the sample at the pump wavelength are reduced by a factor of $\sim 2$. Therefore, neglecting the modifications of the density, the heat capacity and the absorption depth of the sample that occurs during the $\lambda$ - to $\beta$-phase transition, an estimation of the temperature increase $\Delta T$ indicates that it is reduced by a factor of $\sim 4$. Accordingly, upon excitation, the actual temperature increase of the surface of the $\beta-\mathrm{Ti}_{3} \mathrm{O}_{5}$ flakes is in between $250 \mathrm{~K}$ and $500 \mathrm{~K}$. This temperature is here again close to the $\beta$ - to $\alpha$-phase transition. Hence, the surface of the particle is rapidly set in the $\alpha$-phase. As the heat brought by the pump pulse diffuses toward the center, the surface of the particle rapidly cools down and is switched from $\alpha$ - to $\lambda-\mathrm{Ti}_{3} \mathrm{O}_{5}$. Here again one can evaluate the temperature increase at the center of the flake, $\sim 6 \mathrm{~ns}$ after its excitation. It is in between $150 \mathrm{~K}$ and $400 \mathrm{~K}$, below the $\beta$ - to $\alpha$ - phase transition. Therefore the scenario that can accounts for $\beta$ - and $\lambda$-phase transition is as follow: Upon its excitation by nanosecond pump pulse, the temperature at the surface of the particle is above the $\beta$ - to $\alpha$-phase transition. The $\lambda$-domains rapidly grow 

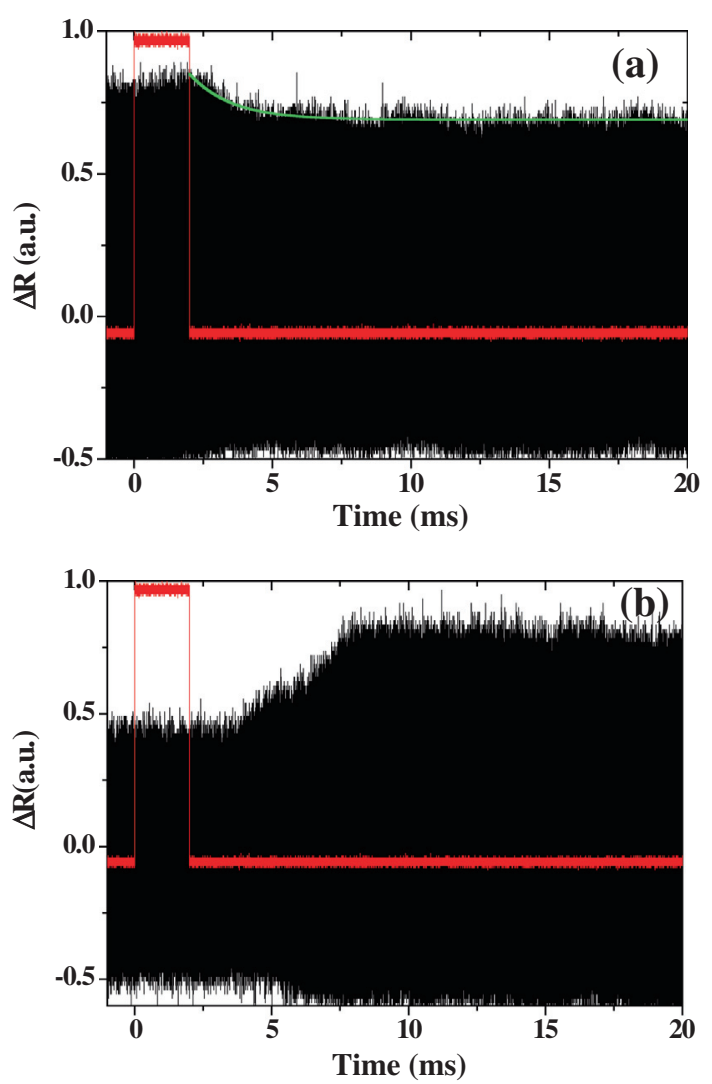

Figure 6. (a) Reflectivity evolution upon application of QCW laser pulse ( $\tau=2 \mathrm{~ms}$, $\lambda=532 \mathrm{~nm}, I=2.410^{-3} \mathrm{~mW} \mu \mathrm{m}^{-2}$ ). The sample is switched from $\lambda$ - to $\beta-\mathrm{Ti}_{3} \mathrm{O}_{5}$. (b) Reflectivity evolution upon application of QCW laser pulse $(\tau=2 \mathrm{~ms}, \lambda=532 \mathrm{~nm}$,

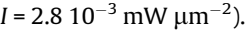

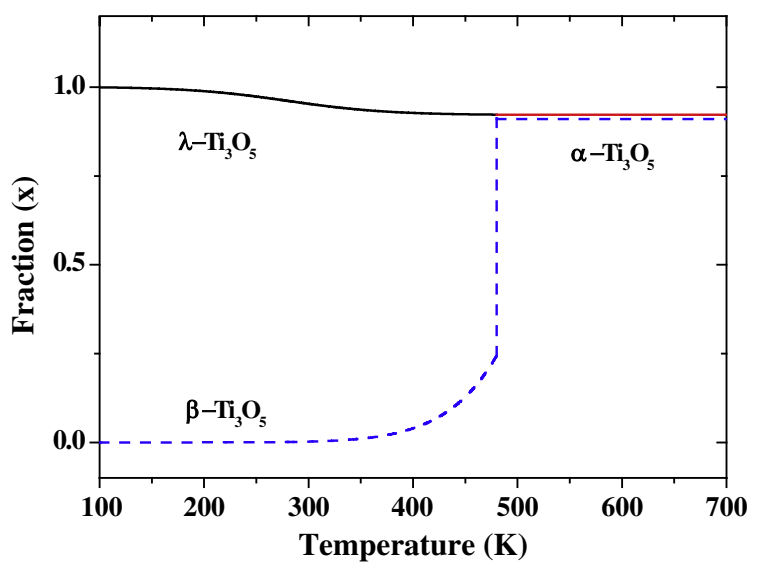

Figure 7. Phase diagram of $\mathrm{Ti}_{3} \mathrm{O}_{5}$ : dotted blue line presents the evolution of the fraction of the $\alpha$-phase versus temperature in bulk sample. The black and red lines represent the evolution of fraction of the $\lambda$ and $\alpha$-phase in nanoparticles versus temperature respectively. (For interpretation of the references to colour in this figure legend, the reader is referred to the web version of this article.)

from the surface towards the center of the particle. However, they are very quickly frozen in as the particles cool down. Hence only a small fraction of the flake close to surface is set in the $\lambda-\mathrm{Ti}_{3} \mathrm{O}_{5}$ phase and others excitation pulses are required to completely switch it in the $\lambda$-phase. Now since the $\alpha$ - to $\lambda$-phase transition is a second order phase transition, it is not accompanied by any latent heat exchange. Growth of the $\lambda-\mathrm{Ti}_{3} \mathrm{O}_{5}$ phase can only occur when the temperature is above $\sim 450 \mathrm{~K}$. This account very well for the shorter characteristic time $(\sim 20 \mathrm{~ns})$ we recorded. Here again, one should consider that this characteristic time is related to the time it takes for the heat to be released from the flake.

The next question we should address is: can the $\lambda$ - to $\beta-\mathrm{Ti}_{3} \mathrm{O}_{5}$ or $\beta$ - to $\lambda-\mathrm{Ti}_{3} \mathrm{O}_{5}$ phase transitions be established on a shorter time scale? The answer to this question is yes [26]. Indeed, since the optical absorption is almost instantaneous, a phase transition for instance from $\beta-\mathrm{Ti}_{3} \mathrm{O}_{5}$ to $\lambda-\mathrm{Ti}_{3} \mathrm{O}_{5}$ resulting from the excitation of the valence band to the conduction band, cannot be discarded. Indeed, once such a transition realized, most of the laser energy absorbed is released as heat induced and results in a large increase of the temperature of sample. Now since our reflectivity signal is integrated over a nanosecond time scale, the large increase of the reflectivity of the sample induced by these thermal effects may hide the smaller reflectivity change induced by the phase transitions [26]. To address properly such photo-induced phase transition, a pump-probe experiment with a sub-picosecond time resolution is required.

Now, we will consider the process taking place for $\lambda$ - to $\beta$-phase transition when QCW pulses are exciting the sample. The evaluation of the temperature increase requires also the knowledge of the particle thermal conductivity. Indeed, at the millisecond time scale, heat must diffuse within, outside the flakes but also in between the flakes that lies beneath the surface of the sample. Then the heat diffusion depends strongly on many parameters such as the contacts between the flakes. However, we can avoid such tedious evaluations by supposing that when a $\mathrm{Ti}_{3} \mathrm{O}_{5}$ particle is heated above $700 \mathrm{~K}$ in ambient air, it turns in $\mathrm{TiO}_{2}$. Figure $3 \mathrm{~b}$ indicates that we do observe such a transformation for pump power density of $2.810^{-3} \mathrm{~mW} \mu \mathrm{m}^{-2}$. Considering very crudely that the temperature scales like the pump power density, one can evaluate that for a power density of $2.410^{-3} \mathrm{~mW} \mu \mathrm{m}^{-2}$, the temperature of the particle is $\sim 640 \mathrm{~K}$. The latter temperature is here again above the $\beta$ - to $\alpha$-phase transition. Accordingly one can consider that as the particles are heated, they are brought in the $\alpha$ phase. As the pump beam is stopped, the particles are rapidly cooled and trapped in $\lambda$-phase. We attributed the $1.5 \mathrm{~ms}$ time scale we recorded in Figure 6 to diffusion of heat from the surface toward the bulk of the sample. Indeed for excitation with QCW pulse lasting $\mathrm{T}$ seconds, heat diffuses over a characteristic length $\delta \sim 2\left(T D_{t h}\right)^{1 / 2}$. If one considers that the sample is excited during $T=2 \mathrm{~ms}$ and has the thermal diffusivity $D_{t h} \sim 410^{-6} \mathrm{~m}^{2} \mathrm{~s}^{-1}$, close to that of $\mathrm{TiO}_{2}$, then one finds that $\delta \sim 180 \mu \mathrm{m}$, length that is large compared the mean radius of the flake $(2 \pm 0.5 \mu \mathrm{m})$.

\section{Conclusions}

In conclusion, by performing single-shot time resolved reflectivity measurements, we have been able to record the characteristic time associated to the reversible $\lambda$ - to $\beta-\mathrm{Ti}_{3} \mathrm{O}_{5}$ phase transition in flakes of $\mathrm{Ti}_{3} \mathrm{O}_{5}$ nanoparticles. We have shown that these phase transitions proceed with different time scales and depend on the duration of the excitation pulses. We proposed a scenario that can well account for the photo-induced phase transitions. In short, according to our scenario, the $\lambda$ - to $\beta-\mathrm{Ti}_{3} \mathrm{O}_{5}$ phase transition induced by nanosecond pulses results both laser induced shock wave and laser induced heating: The laser heating brings the flake above the $\lambda$ - to $\alpha$ - phase transition and the shock wave induces a $\alpha$ - to $\beta$ - phase transition at the surface of the flake. As the flake cools down, the $\beta$-domains grow from the surface toward the center of the flake. For the $\beta$ - to $\lambda-\mathrm{Ti}_{3} \mathrm{O}_{5}$ phase transition our scenario indicates it mainly results from laser induced heating. In this case, since the absorption of the flake and the pulse fluence is lower, the laser heating is lesser. However, the latter is still high enough to bring the surface of the flake above the $\beta$ - to $\alpha$-phase transition. As the surface of the flake cools down, its surface is switched from 
$\alpha$ - to $\lambda$-phase but the center of the flake remains unchanged. Subsequent nanosecond pulses with similar energy density make it possible to switch the whole particle in the $\lambda$-phase. We have also mentioned that $\lambda$ - to $\beta-\mathrm{Ti}_{3} \mathrm{O}_{5}$ or $\beta$ - to $\lambda-\mathrm{Ti}_{3} \mathrm{O}_{5}$ phase transition that can be established on a shorter time scale may be hidden by these thermal processes. Finally for the transition induced by QCW laser, it is the laser induced heating that is responsible for the photoinduced phase transition.

\section{Acknowledgments}

The authors acknowledge support through ANR project RMNP 019-01 'OptoMaTS', Conseil Régional d'Aquitaine via the project 'MatTS', A.O.H. is grateful to the Conseil Régional d'Aquitaine for financial support.

\section{References}

[1] N. Yamada, E. Ohno, K. Nishiuchi, N. Akahira, M. Takao, J. Appl. Phys. 69 (1991) 2849.

[2] A.V. Kolobov, P. Fons, A.I. Frenkel, A.L. Ankudinov, J. Tominaga, T. Uruga, Nat. Mater. 3 (2004) 703.

[3] P. Gütlich, A. Hauser, H. Spiering, Angew. Chem. Int. Ed. 33 (1994) 2024

[4] K. Nasu, Springer, 1997.

[5] O. Kahn, C.J. Martinez, Science 279 (1998) 44.

[6] J.-F. Létard, J.A. Real, N. Moliner, A.B. Gaspar, L. Capes, O. Cador, O. Kahn, J. Am. Chem. Soc. 121 (1999) 10630.
[7] H. Dürr, H. Bouas-Laurent, Elsevier, 1990.

[8] M. Irie, T. Fukaminato, T. Sasaki, N. Tamai, T. Kawai, Org. Nat. 420 (2002) 759.

[9] S. Habuchi, R. Ando, P. Dedecker, W. Verheijen, H. Mizuno, A. Miyawaki, J. Hofkens, Proc. Natl. Acad. Sci. USA 102 (2005) 9511.

[10] S. Ohkoshi, K. Hashimoto, J. Photochem. Photobiol. C 2 (2001) 71.

[11] M. Verdaguer, Science 272 (1996) 698.

[12] S.-I. Ohkoshi, S. Yorozu, O. Sato, T. Iyoda, A. Fujishima, K. Hashimoto, Appl Phys. Lett. 70 (1997) 1040.

[13] J.M. Herrera, V. Marvaud, M. Verdaguer, J. Marrot, M. Kalisz, C. Mathonière, Angew. Chem. Int. Ed. 43 (2004) 5468.

[14] A. Dei, Chem. Int. Ed. 44 (2005) 1160.

[15] S.-I. Ohkoshi, S. Ikeda, T. Hozumi, T. Kashiwagi, K. Hashimoto, J. Am. Chem. Soc 128 (2006) 5320.

[16] H. Tokoro, T. Matsuda, T. Nuida, Y. Moritomo, K. Ohoyama, E.D. Loutete, Chem. Mater. 20 (2008) 423

[17] S. Koshihara, Y. Tokura, T. Mitani, G. Saito, T. Koda, Phys. Rev. B 42 (1990) 6853.

[18] E. Collet, M.-H. Lemée-Cailleau, M. Buron-Le, Science 300 (2003) 612.

[19] K. Miyano, T. Tanaka, Y. Tomioka, Y. Tokura, Phys. Rev. Lett. 78 (1997) 4257.

[20] S.-I. Ohkoshi, Y. Tsunobuchi, T. Madsuda, K. Hashimoto, A. Namai, F. Hakoe, H. Tokoro, Nat. Chem. 2 (2010) 539.

[21] G. Galle, J. Degert, C. Mauriac, C. Etrillard, J.F. Letard, E. Freysz, Chem. Phys. Lett. 500 (2010) 18.

[22] Y. Wu, Q. Zhang, X. Wu, S. Qin, J. Liu, J. Solid State Chem. 192 (2012) 356.

[23] A signal of the same amplitude recorded when the fast photodiode is illuminated by weaker pulse fluence $\left(\sim 110-6 \mathrm{~mJ} \mathrm{\mu m}^{-2}\right)$ only exhibits a signal that lasts few nanoseconds; secondly, this signal keeps its temporal shape when a set of neutral density filters are inserted in front of the fast photodiode.

[24] H.C. Choi, Y.M. Yung, S.B. Kim, Vib. Spectrosc. 37 (2005) 33.

[25] R. Liu, J.-X. Shang, Mater. Sci. Eng. 20 (2012) 035020.

[26] M. Lorenc, Ch. Balde, W. Kaszub, A. Tissot, N. Moisan, M. Servol, M. Buron-Le, Phys. Rev. B. 85 (2012) 054302. 